\title{
Difficulties in determining the dose-response nature of competitive soccer matches
}

\section{Dr Matthew Weston, Teesside University, United Kingdom}

The monitoring of training loads within soccer is now common practice. Training sessions are monitored to evaluate the physical stimulus imposed upon players to ascertain whether the goals of training sessions have been met. An understanding of the demands of training is also required to facilitate the effective planning and timing of subsequent training sessions. Technological advances in the measurement of physical activity - such as GPS and heart rate monitors - enable sport scientists to accurately gauge the actual work performed by their players (i.e., distances covered) and the physiological response to this work (i.e., heart rates). Competitive soccer matches normally occur at the end of the training week and contribute a large percentage of the overall weekly dose of activity. The recent development and introduction of semi-automatic computerised tracking systems, along with GPS measurements, now permits a detailed analytical evaluation of external match load. However, the stimulus for exercise-induced adaptations is the relative physiological stress imposed on athletes (internal load) and not the external load (i.e., the actual work performed) [1]. The collection of physiological data in competitive contexts is limited by restricted access to elite sports performers and the rules and regulations of competitions [2]. Therefore, a lack of internal load measurement during competitive soccer matches makes it difficult to relate external load to internal load and leaves sport scientists unable to quantify the true dose-response nature of matches.

External match loads are very often expressed in terms of the distances covered in selected speed zones (i.e., walking, jogging, running, high-intensity running and sprinting). Such speed zones are demarcated by absolute speed thresholds. Whilst it is the absolute amount of running that contributes most to the match physical performance, arbitrary speed thresholds fail to individualise match activities relative to individual specific movement speeds or physiological capacity $[3,4]$. Without an individualised approach to the measurement of external load, the true energetic demands of a match are unknown [5]. Therefore, in an attempt to overcome this and relate external load to internal load, attempts have been made within the recent literature to individualise speed zones as this will provide a more accurate measure of the dose-response relationship [6]. These studies have provided a useful addition to the literature as they have demonstrated that when speed zones are expressed relative to an individual's physical capacity (either maximal running speed or laboratory-determined physiological thresholds [i.e, $\left.\mathrm{VT}_{2}\right]$ ) this provides a more accurate reflection of the physical stimulus imposed during a soccer match.

Whilst this individualised approach represents a sound attempt to elucidating the dose-response nature of soccer matches by relating external and internal loads, methodological difficulties in this approach have yet to be fully addressed. For example, the application of physiological thresholds derived from laboratory-based continuous exercise protocols to intermittent exercise is questionable, especially 
given the stochastic environment that is a competitive soccer match. If match activity zones are to be calculated from maximal running speeds difficulty could lie in the classification (as a percentage of maximal speed), description and justification of the different match activity zones [7]. Furthermore, the facilities and expertise required for the determination of individualised speed zones may prove to be restrictive [5].

The measurement and evaluation of internal match loads, in particular ratings of perceived exertion, alongside match running performances could help to accurately relate match activities to the physical capacity in an attempt to understand the dose-response nature of competitive soccer matches. Ratings of perceived exertion (RPE) are a practical, non-invasive and accurate means of monitoring exercise intensity, with concurrent validity being evidenced via strong relationships with other indices of exercise intensity [1,8-10]. Ratings should refer to overall perceived exertion, which depends on many factors that are integrated into a kind of gestalt or configuration [11] and the scores should be therefore representative of both internal and external load $[1,10]$. However, the limited data that are available have demonstrated competitive soccer match RPE's to be stable measures in both players [12] and referees [13]. The lack of variability of these measures during competitive matches is of concern when attempting to understand the dose-response nature of matches given the high variability reported for measures of external load in soccer, in particular high-speed running and sprinting $[3,4]$. When interpreting competitive match heart rates, which is practical in soccer referees, a similar phenomenon is apparent in that the measure of internal load is far more stable when compared to measures of external load. Therefore, whilst these measures of internal load may provide a global measure of match intensity they appear to lack sensitivity to accurately relate to measures of external load.

A comprehensive evaluation of external and internal training loads in soccer is now possible given the measurement tools that are now available to the sport scientist. Whilst more comprehensive data are now available for the evaluation of external match loads via the introduction of semi-automatic computerised tracking systems, the rules and regulations of competitive matchplay limit the collection of internal match loads. Consequently, an in-depth understanding of the dose-response nature of matches is difficult and this could be detrimental to the effective planning and timing of subsequent training sessions. Individualised speed zones represent a logical attempt to relate external loads to internal loads, although this approach can be expensive and time-consuming with methodological flaws that have yet to be addressed within the literature. Given the restricted access to players during matches, global ratings of perceived exertion provide an attractive solution in the attempt to relate external loads to internal loads. However, given such global ratings of perceived exertion appear to lack the sensitivity to account for the varying physical match demands. Differential ratings of perceived exertion (i.e., separate ratings for physical and technical match demands) may discriminate 
the highly variable external physical loads, thereby providing a more sensitive evaluation of match internal load.

\section{References}

1. Impellizzeri FM, Rampinini E, Coutts AJ, Sassi A, Marcora SM (2004) Use of RPE-based training load in soccer. Med Sci Sports Exerc 36: 1042-1047.

2. Drust B, Atkinson G, Reilly $T$ (2007) Future perspectives in the evaluation of the physiological demands of soccer. Sports Med 37: 783-805.

3. Gregson W, Drust B, Atkinson G, DiSalvo V (2010) Match-to-match variation of high-speed activities in Premier League soccer. Int J Sports Med 31: 237-242.

4. Weston M, Drust B, Atkinson G, Gregson W (2011) Variability of soccer referees' match performances. Int J Sports Med 32: 190-194.

5. Lovell R, Abt G (2012) Individualisation of time-motion analysis: a case-cohort example. Int J Sports Physiol Perform, in press

6. Abt G, Lovell R (2009) The use of individualized speed and intensity thresholds for determining the distance run at high-intensity in professional soccer. J Sports Sci 27: 893-898.

7. Weston M, Castagna C, Impellizzeri FM, Bizzini M, Williams AM, et al. (2012) The science and medicine applied to soccer refereeing: an update. Sports Med 42: 615-631.

8. Foster C (1998) Monitoring training in athletes with reference to overtraining syndrome. Med Sci Sports Exerc 30:1164-1168.

9. Foster C, Florhaug JA, Franklin J, Gottshcall L, Hrovation LA et al (2001) A new approach to monitoring exercise training. J Strength Cond Res 15:109-115.

10. Coutts AJ, Rampinini E, Marcora SM, Castagna C, Impellizzeri FM (2009) Heart rate and blood lactate correlates of perceived exertion during small-sided soccer games. J Sci Med Sport 21: 79-84.

11. Borg G (1998) Borg's perceived exertion and pain scales. Human Kinetics Publishers.

12. Wrigley R, Drust B, Stratton G, Scott M, Gregson W (2012) Quantification of the typical weekly in-season training load in elite junior soccer players. J Sports Sci 30: 1573-1580.

13. Weston M, Bird S, Helsen W, Nevill A, Castagna C (2006) The effect of match standard and referee experience upon the objective and subjective match workload of English Premier League referees. J Sci Med Sport 9: 256-262. 